\title{
Mitigating the Impact of COVID-19 on Funded Clinical Research: Crucial Next Steps
}

\author{
Rebekah J. Walker, $P h D^{1,2}$, Jeffrey L. Jackson, MD, MPH ${ }^{1,3}$, Steven M. Asch, MD, MPH ${ }^{4,5}$, \\ and Leonard E. Egede, MD, MS ${ }^{1,2}$
}

'Division of General Internal Medicine, Department of Medicine, Medical College of Wisconsin, Milwaukee, WI, USA; ${ }^{2}$ Center for Advancing Population Science, Medical College of Wisconsin, Milwaukee, WI, USA; ${ }^{3}$ Clement J. Zablocki Veterans Affairs Medical Center, Milwaukee, WI, USA; ${ }^{4}$ Center for Innovation to Implementation (Ci2i), Veteran Affairs Palo Alto Health Care System, Palo Alto, CA, USA; ${ }^{5}$ Division of Primary Care and Population Health, Stanford University, Stanford, CA, USA.

J Gen Intern Med 36(2):518-20

DOI: $10.1007 / \mathrm{s} 11606-020-06342-8$

(c) Society of General Internal Medicine 2020

\section{PERSPECTIVE}

The COVID-19 pandemic is a catastrophic event that has resulted in unprecedented disruption of clinical research and could have grave long-term consequences on the integrity and rigor of funded research. Clinical research improves lives and has a significant impact on economic productivity, and advancement of science, worldwide. ${ }^{1,2}$ Scientific progress relies on systematic and continuous funding of innovative and transformative ideas, and requires dedication to funding both edge science (the regular exercise of trying out new ideas) and high impact science. ${ }^{3,4}$ The initial phase of the COVID-19 pandemic shutdown much of clinical research through quarantines, site closures, social distancing restrictions, travel limitations, interruptions in the supply chain, and increased risk to both personnel and study participants. ${ }^{5}$ Recovery and reopening has been slow due to a shortage of consistent access to personal protective equipment (PPE), limits on the size of gatherings impacting recruitment and consent procedures, the need for staff to quarantine after suspected exposure due to limited rapid test options, and impacts on transportation and stability of many research participants.

Though some flexibilities in federal clinical research spending were provided through the end of September $2020^{6-8}$, the resurgence of COVID-19 in many regions and difficulties in restarting research, particularly randomized controlled trials, make it unlikely that the majority of clinical research will meet pre-COVID milestones and deadlines for recruitment. In addition, many funding agencies and research institutions are focused on prioritizing COVID-19 research studies, although the public health challenges and clinical problems that existed before COVID-19 are still very relevant. Important research questions on chronic disease, cancer, drug development, and aging, for example, will remain even after a vaccine is

Received August 3, 2020

Accepted October 31, 2020

Published online November 17, 2020 developed for COVID-19. Therefore, it is necessary to protect non-COVID-19 focused clinical research.

There are four primary challenges facing ongoing nonCOVID-19-related research that will require flexibility in approach, timelines, and targets to ensure research integrity. First, disruption of research studies and changes in safe procedures for recruiting and monitoring participants will require accommodations by research teams. Second, research participants now face additional challenges and considerations, which will require accommodations specific to their needs. Third, there will be difficulty in completing funded research projects within original timelines. Funders, including the National Institute of Health (NIH), Department of Veterans Affairs (VA), Department of Defense (DoD), Federal Drug Administration (FDA), pharmaceutical companies, and funding agencies worldwide will need to work with research teams to identify and accommodate changes identified as necessary to maintain integrity of research studies. And finally, there will be challenges in timely publication of non-COVID trial results, which can be facilitated by journals and editors. Table 1 provides a summary of suggestions to address each of these challenges with further details below.

\section{Accommodations for Research Teams}

Investigators have published a variety of strategies to address the impact of COVID-19, including study modifications, revision of protocols, modified or adaptive designs, and statistical analysis modifications. ${ }^{9-14}$ Changes in how participants can safely be engaged in studies may require data collection using electronic data capture or telephone interviews. ${ }^{9,13}$ Studies unable to use remote study visits should combine or reduce the number of essential in-person visits, and studies using sites with large numbers of individuals should identify alternative locations that are less crowded. ${ }^{10,14}$ To ensure transparency in changes to the study and allow for consideration during statistical analysis, study staff should document when participation in the study was affected by COVID-19, and dates and reasons for changes in data collection., ${ }^{9} 11$ Given staff disruption is possible as individuals quarantine after suspected exposure, organizing virtual daily staff "huddles" can be used to 
Table 1 Recommendations for Accommodations to Ensure Integrity of Non-COVID Research, Completion of Non-COVID Studies, and Timely Publication of Non-COVID Trial Results

Accommodations for research teams to ensure research integrity

1. Use electronic data capture or telephone interviews to minimize inperson visits.

2. Combine or reduce the number and length of essential in-person visits and use locations that are less crowded.

3. Document how participation in the study was affected by COVID19 , including dates and reasons for changes in data collection procedures.

4. Organize daily staff check-in to discuss operational challenges and promote consistent procedures.

5. Critically examine methods and frequency of protocolized safety assessments.

6. Discuss how to handle trial data collected during disruption, and document changes in the statistical analysis plan prior to completion of study.

7. Consider additional analyses including center-by-treatment interactions, time trends, and cohort effects.

Accommodations for research participants to address additional challenges

1. Adjust compensation for participants to take into account additional time and modifications to daily life needed to participate.

2. Provide flexibility in visits, such as offering hybrid visits with only biospecimen collection requiring in-person visits.

3. Consider changes in transportation options for participants, such as providing home visits.

4. Incorporate community advisory boards and stakeholder engagement activities as part of research recovery.

5. Consider use of home telemonitoring devices to capture clinical outcomes.

Accommodations for funders to facilitate completion of studies

1. Give program officers the authority to approve reduction in or changes in scope of funded research without penalty.

2. Relax research progress reporting requirements, specifically for milestone plans in clinical trials.

3. Provide administrative supplements to support changes necessary to accommodate COVID-19.

4. Consider extensions with cost for 12-months after the original end date of funded projects.

5. Extend deadlines for completion of clinicaltrials.gov reporting and timelines for depositing of data to allow completion of additional analyses.

6. Build in reporting on impact of COVID-19 either through the final report or clinicaltrials.gov.

Accommodations for journals and editors to facilitate timely publication

1. Set reasonable expectations to ensure that rigor is maintained in reporting of results from clinical trials impacted by COVID-19 but be realistic and flexible during review.

2. Accept documentation of changes shown through IRB amendment approvals indicating changes in procedures or changes to statistical analysis plans.

3. Encourage clinical trials to discuss how COVID19 impacted the integrity and validity of results and provide insight into the impact of COVID-19 on their findings.

discuss operational challenges and promote consistent procedures. ${ }^{13}$ To ensure all staff are following standard safety procedures that account for new COVID-19-specific considerations, methods and frequency of protocolized safety assessments should be critically examined. ${ }^{14}$ Given that changes to the initially planned analysis may be necessary, investigators should discuss how to handle trial data collected during disruption prior to study completion. Changes in the statistical analysis plan should be documented through a protocol amendment and provided to journals at the time of manuscript submission. ${ }^{9}$ Additional analyses may include investigation for center-by-treatment interactions, time trends, cohort effects, possible spurious inflation of events, or decrease in rates due to changes in health care access. ${ }^{12}$

\section{Accommodations for Research Participants}

Research participants may have significant disruption in their daily lives, including loss of employment, so will require accommodation. First, it may be necessary to increase compensation to participants, which may require rebudgeting or supplemental funding. Second, study visit flexibility may be necessary. Hybrid of in-person and virtual visits where surveys can be conducted virtually and biospecimen collection in-person may be needed. For teams that decide to use webbased, electronic data capture tools, consideration should be given to potential negative impact on vulnerable populations and pros and cons of these challenges will need to be factored into decisions. Third, transportation options may be limited due to disruptions from the pandemic; therefore, alternatives like offering home visits using appropriate safety precautions should be considered. Fourth, given the multiple layers of disruption from the pandemic, study teams should consider convening community advisory boards to help them tailor recruitment, retention, and study completion strategies to match the unique needs of their population. Finally, consideration should be given to using home telemonitoring devices to collect clinical outcomes data with appropriate calibration.

\section{Accommodations for Funders}

Several accommodations by funders may be necessary to mitigate the impact of the pandemic and ensure maximum flexibility for research teams, while maintaining the rigor of funded research. These accommodations need to be transparent and consistently applied across programs. We propose a series of accommodations in a 12-month cycle based on experience and feedback from investigators. Within 12 months of studies being reactivated, program officers should have the flexibility to approve reductions or changes in the scope of funded research without penalty to the investigative team. The scope of previously funded research should be reevaluated within the reality of COVID-19 and necessary modifications should be determined as a collaborative process. These modifications may include changes in study aims, modifications to intervention delivery, or changes in study sites. Additional modifications may include changes to intervention procedures such as smaller group sessions, virtual intervention delivery, use of home telemonitoring devices for capturing outcome measures, and consideration of electronic health records for primary outcome data capture when feasible. Secondly, relaxed research progress reporting requirements may be necessary to minimize penalties incurred for not meeting pre-COVID timelines. This will be particularly important for NIH milestone plans for clinical trials, which tie funding continuation to recruitment and retention goals set prior to the pandemic.

Between 12 and 24 months following research activation, investigators should have assessed the true needs of their study and may need the ability to request administrative supplements for non-COVID-19 studies. Many NIH institutes and 
other US federal funding agencies currently offer administrative supplements; however, most are specific to COVID-19 research conducted in addition to the funded study. Administrative supplements may be needed to support changes necessary to accommodate COVID-19, such as the need to hire more personnel, increase incentives or provide transportation for participants, purchasing of PPE or other changes necessary to ensure safety of study participants and research personnel, or the cost of adding new sites to existing studies.

Then, between 24 and 36 months following research activation, consideration should be given to approving a 12month extension with cost for studies that do not have adequate time left to meet their pre-pandemic study timelines. Given the slow process for restarting recruitment, additional time may be needed to finish with the appropriate sample size, and therefore sufficient power, to answer their primary research question. In addition, requirements for completion of clinicaltrials.gov reporting or depositing data within 12 months of study completion should be extended to allow additional analyses that investigate the impact of COVID-19 on findings. Finally, funders should build in new reporting requirements that include a report of the impact of COVID-19 on recruitment, retention, study completion, and power, and investigators should be encouraged to report similar data in clinicaltrials.gov for clinical trials.

\section{Accommodations for Journals and Editors}

Lastly, it will be necessary for editors of peer-reviewed journals to set reasonable expectations to ensure that rigor is maintained in reporting of results from clinical trials impacted by COVID-19 but be realistic and flexible during review. Clinical trials should be required to report the specific amendments made to modify recruitment methods, intervention delivery, or statistical analyses. As many studies have already published protocol papers, journals should accept documentation of changes shown through IRB amendment approvals indicating changes in procedures or changes to statistical analysis plans. Editors and reviewers should be flexible and realistic in evaluating studies forced to reduce sample size or modify intervention delivery. Journal editors should also encourage authors of clinical trials papers to discuss how COVID-19 impacted the integrity and validity of results and provide insight into the impact of COVID-19 on their findings. This may be done through secondary analyses using such approaches as interrupted time series that look at the disruptive effect of COVID-19, or through more qualitative discussion of the unique and unexpected challenges experienced and how this may have influenced recruitment, retention, intervention delivery, and study results.

Clinical research has substantially advanced the health and economic wellbeing of the global population. Given the wide ranging effects of COVID-19 pandemic on investigatorinitiated clinical research, a series of accommodations by research teams, research participants, funders, and journals may be necessary to mitigate the impact of the pandemic and ensure maximum flexibility for research team, while maintaining rigor of funded research.

Corresponding Author: Leonard E. Egede, MD, MS; Division of General Internal Medicine, Department of Medicine, Medical College of Wisconsin, Milwaukee, WI, USA (e-mail: legede@mcw.edu).

\section{Compliance with Ethical Standards:}

Conflict of Interest: The authors declare that they have no competing interests. Effort for authors was partially supported by the National Institute of Diabetes and Digestive Kidney Disease (K24DK093699, R01DK118038, R01DK120861, PI: Egede), the National Institute for Minority Health and Health Disparities (R01MD013826, PI: Egede/ Walker), and the American Diabetes Association (1-19-JDF-075, PI: Walker. Content of this article is solely the responsibility of the authors and does not necessarily represent the official views of the National Institutes of Health.

\section{REFERENCES}

1. National Institute of Health (NIH). Impact of NIH Research. Available at: https://www.nih.gov/about-nih/what-we-do/impact-nih-research.

2. Murphy KM, Topel RH. The Value of Health and Longevity. U Chicago and National Bureau of Economic Research. 2006. Available at: https:// ucema.edu.ar/u/je49/capital_humano/Murphy_Topel_JPE.pdf. Accessed 11 Nov 2020.

3. Packalen M, Bhattacharya J. NIH funding and the pursuit of edge science. PNAS. 2020; 117(22): 12011-12016.

4. Moses H, Matheson DHM, Cairns-Smith S, George BP, Palisch C, Dorsey ER. The Anatomy of Medical Research: US and International Comparisons. JAMA. 2015; 313(2): 174-189.

5. US Dept of Health and Human Services, Food and Drug Administration (FDA). FDA Guidance on Conduct of Clinical Trials of Medical Products during COVID-19 Public Health Emergency: Guidance for Industry, Investigators, and Institutional Review Boards. July 2, 2020.

6. M 20-26. Extension of Administrative Relief for Recipients and Applicants of Federal Financial Assistance Directly Impacted by the Novel Coronavirus (COVID-19) due to Loss of Operations. June 18, 2020.

7. Mervis J. New White House rules restrict use of grant funding to deal with COVID-19 impacts. Science. June 30, 2020. Available at: https:// www.sciencemag.org/news/2020/06/new-white-house-rules-restrictuse-grant-funding-deal-covid-19-impacts. Accessed 11 Nov 2020.

8. National Institute of Health (NIH). NIH Implementation of OMB Memorandum M-20-26 "Extension of Administrative Relief for Recipients and Applicants of Federal Financial Assistance Directly Impacted by the Novel Coronavirus (COVID-19) due to Loss of Operations" dated June 18, 2020. Available at: https://grants.nih.gov/policy/natural-disasters/corona-virus/nih-omb-memo.htm. Accessed 11 Nov 2020.

9. Fleming TR, Labriola $\mathbf{D}$, Wittes J. Conducting clinical research during the COVID-19 pandemic: protecting scientific integrity. JAMA. 2020; 324(1): 33-34

10. Best Practices for Conducting Trials During the COVID-19 Pandemic. Clinical Trials Transformation Initiative (CTTI).

11. Gidley J, Grignolo A, Heelan B, McKee A. Five strategies for mitigating the impact of COVID-19 on clinical trials. Endspoints News. Available at: https://endpts.com/sp/five-strategies-for-mitigating-the-impact-ofcovid-19-on-clinical-trials/. Accessed 11 Nov 2020.

12. Bagiella E, Bhatt DL, Gaudino M. The consequences of the COVID-19 pandemic on non-COVID-19 clinical trials. J Am Coll Cardiol. 2000; 76(3):342-345.

13. Waterhouse DM, Harvey RD, Hurley P, Levit LA, Kim ES, et al. Early impact of COVID-19 on the conduct of oncology clinical trials and longterm opportunities for transformation: findings from an American Society of Clinical Oncology Survey. JCO Oncol Pract. 2020; 16: 417-421.

14. Doherty GJ, Goksu M, de Paula BHR. Rethinking cancer clinical trials for COVID-19 and beyond. Nat Cancer. 2020; 1:568-572.

Publisher's Note: Springer Nature remains neutral with regard to jurisdictional claims in published maps and institutional affiliations. 